\title{
Exploring CCD Camera Parameters with Off-Axis Electron Holography
}

\author{
K.J. Dudeck*, E. Voelk1** and G.A. Botton* \\ * Department of Materials Science and Engineering, McMaster University, 1280 Main St W., \\ Hamilton, ON L8S 6L7, Canada \\ ** FEI Company, 5350 NE Dawson Creek Drive, Hillsboro, OR 97124-5793
}

Off-axis electron holography is a powerful technique that allows for the phase and amplitude of the electron wave function to be reconstructed from single transmission electron microscope (TEM) images recorded with the aid of an electrostatic biprism. The complex reconstructed data can yield quantitative information about the specimen, including electric and/or magnetic field distributions and variations in structure or composition [1]. The final resolution of the reconstructed phase (or amplitude) is governed by both experimental and reconstruction parameters; modern microscopes can provide experimental reconstructions with phase resolutions of $2 \pi / 1000$ [2].

Recent work has investigated the effect of experimental and reconstruction parameters on phase- and amplitude-noise, primarily from a theoretical basis via simulations [3]. One of the key elements that define the phase- and amplitude-noise is the number of electrons contributing to the hologram. However, this number is not easily extracted from an experimentally obtained digital image. In Gatan's DigitalMicrograph, a conversion factor is used to convert the experimental pixel values, $P_{m, n}^{e}$ with $m, n$ defining the pixel position, into electron counts, $P_{m, n}$. This approach implies a simple correlation between $P_{m, n}^{e}$ and $P_{m, n}$ according to:

$$
P_{m, n}=\frac{P_{m, n}^{e}}{g}
$$

with the parameter $g$ being the conversion factor. It is important to note the $g$ actually does not impact the noise in the reconstructed phase/amplitude, noise measured as normalized standard deviation for the amplitude and as standard deviation for the phase. However, in order to compare the simulated noise data with the experimental data, it is necessary to determine $g$. Only then can the camera parameters that increase the amplitude- and phase-noise in the reconstructed data be evaluated.

As discussed in [3], the conversion factor $g$ can, e.g., be derived from two consecutive holograms with no object as:

$$
g \leq \frac{\sigma^{2} \mu^{2} M^{2}\left\langle P_{m, n}^{e}\right\rangle}{r^{2} \pi 2^{j+1}}
$$

where $\sigma$ is the phase- or (normalized) amplitude-noise, $\mu$ is the fringe contrast, $M$ is the number of pixels in the reconstruction, $r$ is the radius of the reconstruction aperture and $j$ is either 1 or 0 depending on whether or not a reference image has been used (see Ref. [3] for discussion).

In this work, we consider if and how $g$ varies as a function of the experimental and/or reconstruction parameters, i.e., incident current density, fringe spacing and size of reconstruction aperture. Thus we can explore the effect of the CCD camera on electron holograms recorded at a variety of different conditions, focusing on how the number of counts in the hologram relate to the number of electrons recorded. For example, Fig. 1 presents a plot showing how $g$ varies as a function of the fringe 
sampling $s$, where $s$ is defined as the number of pixels per fringe, for three different values of $\left\langle P_{m, n}^{e}\right\rangle$ (CCD binning factor 2). Fig. 2 presents a plot of $g$ as a function of $s$ for different CCD camera binning factors and constant $\left\langle P_{m, n}^{e}\right\rangle$ (note that the data shown in Figs. 1 and 2 were collected on different microscopes using the same type of CCD camera) [4].

\section{References}

[1] M.R. McCartney and D.J. Smith, Annual Reviews in Materials Research 37 (2007) 729.

[2] E. Voelkl and D. Tang, Ultramicroscopy 110 (2010) 447.

[3] E. Voelk1, Ultramicroscopy 110 (2010) 199.

[4] We are grateful to the Ministry of Research and Innovation of Ontario for financial support (KJD) and NSERC for a Discovery Grant (GAB).
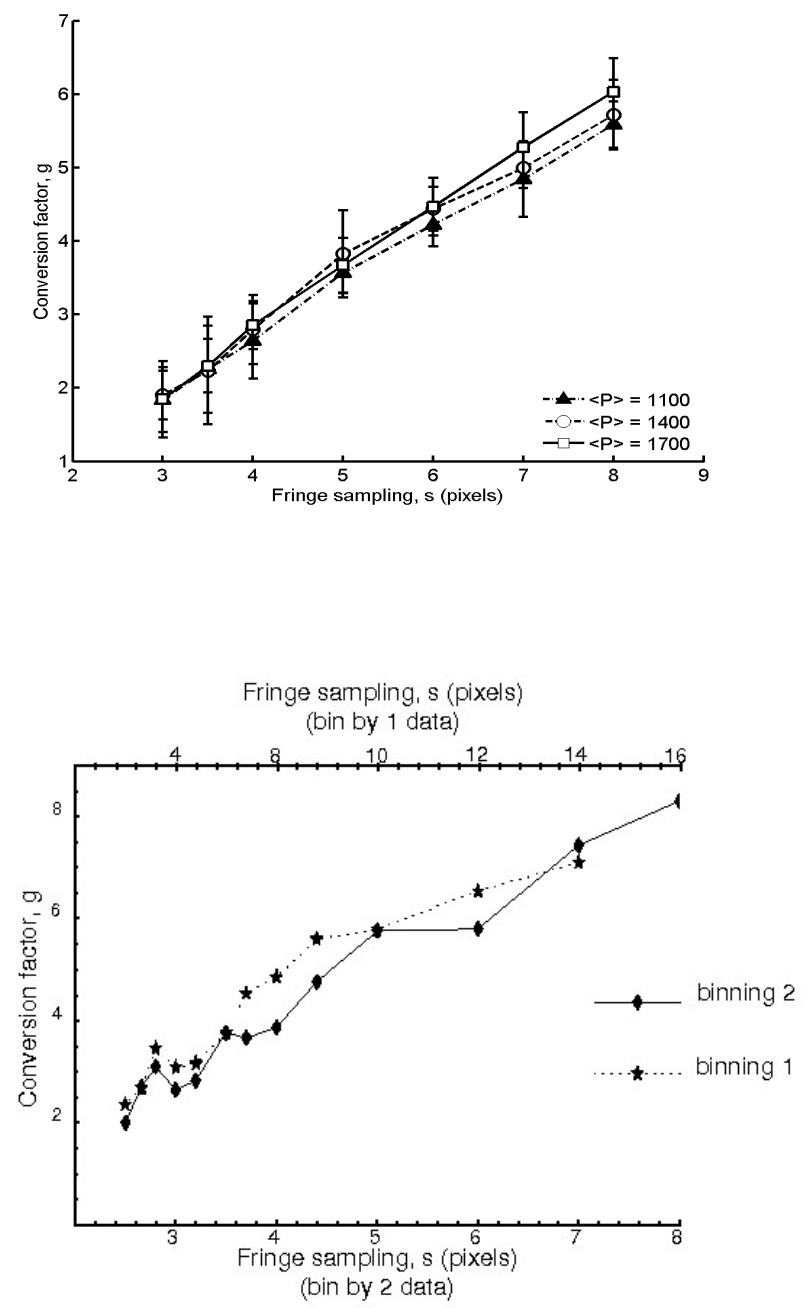

Fig. 1: Conversion factor between image counts and electrons, $g$, as a function of fringe sampling, $s$, for different mean image counts, $\left\langle P_{m, n}^{e}>\right.$ (binning 2$)$.
Fig. 2: Conversion factor between image counts and electrons, $g$, as a function of fringe sampling, $s$, for different binning (constant $<P_{m, n}^{e}>$ ). 